\title{
Local Minimal Curves in Homogeneous Reductive Spaces of the Unitary Group of a Finite von Neumann Algebra
}

\author{
Eduardo Chiumiento
}

To my family

\begin{abstract}
We study the metric geometry of homogeneous reductive spaces of the unitary group of a finite von Neumann algebra with a non complete Riemannian metric. The main result gives an abstract sufficient condition in order that the geodesics of the Levi-Civita connection are locally minimal. Then, we show how this result applies to several examples.
\end{abstract}

Mathematics Subject Classification (2000). Primary 58B20; Secondary 46L10. Keywords. Finite von Neumann algebra, metric geometry, Finsler metric.

\section{Introduction}

The aim of this work is to study local minimal curves in homogeneous reductive spaces of the unitary group of a finite von Neumann algebra, where we define a Riemannian metric in terms of the reductive structure and the trace of the algebra.

Let $\mathcal{M}$ be a finite von Neumann algebra with a fixed trace $\tau$. Denote by $\mathcal{M}_{a h}$ the real Banach subspace of antihermitic elements of $\mathcal{M}$, which identifies with the Lie algebra of the unitary group $U_{\mathcal{M}}$ of the algebra. Let $L^{2}(\mathcal{M}, \tau)$ be the completion of $\mathcal{M}$ with the norm $\|x\|_{2}=\tau\left(x^{*} x\right)^{1 / 2}$. Consider $\mathcal{P}$ an (eventually infinite dimensional) homogeneous reductive space of $U_{\mathcal{M}}$. For each $\rho \in \mathcal{P}$, there is defined in the tangent space $(T \mathcal{P})_{\rho}$ the coordinate map $K_{\rho}$ of the reductive structure taking values on $\mathcal{M}_{a h}$. Then, we can introduce a metric: for $X \in(T \mathcal{P})_{\rho}$,

$$
\|X\|_{\rho}=\left\|K_{\rho}(X)\right\|_{2} \text {. }
$$

In general, this quadratic metric does not induce a complete norm in the tangent spaces. The homogeneous spaces treated here fit in the context of what is usually known as weak Riemannian manifolds (see [15]). Therefore the classical theory of 
Riemann-Hilbert manifolds is not available, so it makes sense to ask about the local minimality of the geodesics. We give a sufficient condition to demonstrate a result on minimality of geodesics of the Levi-Civita connection in the general setting of homogeneous reductive spaces: $\tau$-orthogonality of the supplements in $\mathcal{M}_{a h}$ with the Lie algebras of the structure groups.

Metric geometry in homogeneous spaces in the context of operator algebras is an area of current research. We can cite the remarkable works [8], [9] of C. Durán, L. Mata-Lorenzo and L. Recht, where they study the problem of finding minimal curves with a quotient metric induced by the operator norm. In the case of a finite algebra, metric and differentiable properties of several examples are treated in [1], [3], [7] and the references given there.

The contents of the paper are the following. Section 2 contains basic facts about homogeneous reductive spaces and the metric. In section 3 we state and prove our main result concerning the minimality of the geodesics. In section 4 we give examples of homogeneous spaces satisfying the orthogonality condition. This condition naturally arises in several homogeneous spaces related to operator algebras: unitary orbits of states, normal elements, spectral measures, *homomorphisms, conditionals expectations and partial isometries. Finally, we give an example of an homogeneous space related to some particular $C^{*}$-dynamical systems.

\section{Homogeneous reductive spaces}

We recall the definition of homogeneous reductive spaces and establish basic facts. For a deeper discussion of homogeneous reductive spaces we refer the reader to [6], [12] and [13]. In this work by a homogeneous reductive space we mean:

- A $C^{\infty}$ Banach manifold $\mathcal{P}$.

- A smooth transitive action of the unitary group $U_{\mathcal{M}}$ on $\mathcal{P}$. We denote it by $L_{u} \rho$, where $u \in U_{\mathcal{M}}, \rho \in \mathcal{P}$.

- For each $\rho \in \mathcal{P}$ the map

$$
\Pi_{\rho}: U_{\mathcal{M}} \longrightarrow \mathcal{P}, \Pi_{\rho}(u)=L_{u} \rho
$$

is a principal bundle with structure group $G_{\rho}=\left\{u \in U_{\mathcal{M}}: L_{u} \rho=\rho\right\}$ (called the isotropy group of $\rho$ ).

- There is a smooth distribution of horizontal spaces $\left\{\mathcal{H}_{\rho}: \rho \in \mathcal{P}\right\}$ which are supplements for the Lie algebra of $G_{\rho}$ :

$$
\mathcal{H}_{\rho} \oplus \operatorname{Lie}\left(G_{\rho}\right)=\mathcal{M}_{a h}, \quad \rho \in \mathcal{P} .
$$

These supplements are invariant under the inner action of $G_{\rho}$ :

$$
u \mathcal{H}_{\rho} u^{*}=\mathcal{H}_{\rho}, \quad \rho \in \mathcal{P}, u \in G_{\rho} .
$$

From now on $\mathcal{P}$ stands for a homogeneous reductive space. We call a homogeneous reductive space $\mathcal{P}$ orthogonal if for each $\rho \in \mathcal{P}$ the supplements $\mathcal{H}_{\rho}$ are $\tau$-orthogonal to $\operatorname{Lie}\left(G_{\rho}\right)$. For brevity, we shall frequently just say that $\mathcal{P}$ is orthogonal. 
Remark 2.1. Let us denote by $L^{2}\left(\mathcal{M}_{a h}, \tau\right)$ the real Hilbert space obtained by completion of $\mathcal{M}_{a h}$ in the 2-norm. One can easily check that if $\mathcal{P}$ is orthogonal, then for all $\rho \in \mathcal{P}$ the orthogonal projection

$$
\mathbf{P}_{\rho}: \mathcal{M}_{a h} \longrightarrow \overline{\operatorname{Lie}\left(G_{\rho}\right)} \|^{\|}
$$

satisfies $\mathbf{P}_{\rho}\left(\mathcal{M}_{a h}\right) \subseteq \operatorname{Lie}\left(G_{\rho}\right)$.

Moreover, the converse is also true in the following sense: if the above projection preserves bounded elements for some $\rho \in \mathcal{P}$ (and hence for all), then $\mathcal{H}_{\rho}:=\left(I-\mathbf{P}_{\rho}\right)\left(\operatorname{Lie}\left(G_{\rho}\right)\right)$, defines a smooth distribution of norm closed supplements of $\operatorname{Lie}\left(G_{\rho}\right)$ which are invariant under the inner action of $G_{\rho}$. Indeed, the distribution is smooth since

$$
\mathbf{P}_{L_{u} \rho}=A d(u) \circ \mathbf{P}_{\rho} \circ \operatorname{Ad}\left(u^{*}\right),
$$

where $A d(u): \mathcal{M} \longrightarrow \mathcal{M}, A d(u)(x)=u x u^{*}$. In order to show that each $\mathcal{H}_{\rho}$ is norm closed, let $x_{n}$ be a sequence in $\mathcal{H}_{\rho}$ such that $\left\|x_{n}-x\right\| \rightarrow 0$, then

$$
\left\|\left(I-\mathbf{P}_{\rho}\right)(x)-x_{n}\right\|_{2} \leq\left\|x-x_{n}\right\|_{2} \leq\left\|x-x_{n}\right\| \rightarrow 0 .
$$

Therefore, $\left(I-\mathbf{P}_{\rho}\right)(x)=\lim x_{n}=x$, so we obtain $x \in \mathcal{H}_{\rho}$. On the other hand, the invariance of the supplements is automatically verified. We have

$$
\mathcal{H}_{\rho}=\left\{y \in \mathcal{M}_{a h}: \tau(y x)=0, \forall x \in \operatorname{Lie}\left(G_{\rho}\right)\right\} .
$$

It is easily seen that $u^{*} \operatorname{Lie}\left(G_{\rho}\right) u=\operatorname{Lie}\left(G_{\rho}\right)$, for all $u \in G_{\rho}$. Therefore, we obtain for $y \in \mathcal{H}_{\rho}, x \in \operatorname{Lie}\left(G_{\rho}\right)$,

$$
\tau\left(\left(u y u^{*}\right) x\right)=\tau\left(y\left(u^{*} x u\right)\right)=0 .
$$

Thus, uyu* $\in \mathcal{H}_{\rho}$, and our assertion is proved.

Now we introduce the Riemannian metric as follows. The differential at the identity of $\Pi_{\rho}$ gives the map $\delta_{\rho}:=\left(d \Pi_{\rho}\right)_{1}: \mathcal{M}_{a h} \longrightarrow(T \mathcal{P})_{\rho}$ that induces the isomorphism

$$
K_{\rho}:=\left[\delta_{\rho}\right]^{-1}:(T \mathcal{P})_{\rho} \longrightarrow \mathcal{H}_{\rho} \text {. }
$$

We endow $\mathcal{P}$ with an inner product on each tangent space, in order that these maps become isometric isomorphisms, i.e. for $X, Y \in(T \mathcal{P})_{\rho}$,

$$
\langle X, Y\rangle_{\rho}=\tau\left(K_{\rho}(Y)^{*} K_{\rho}(X)\right) .
$$

Therefore,

$$
\|X\|_{\rho}=\left\|K_{\rho}(X)\right\|_{2}
$$

It is apparent that this defines an invariant metric under the action of $U_{\mathcal{M}}$

Remark 2.2. Since $\mathcal{P}$ is a homogeneous space i.e. a quotient, it would be natural to put in $\mathcal{P}$ a quotient metric. One can define it by means of the 2-norm: for $X \in(T \mathcal{P})_{\rho}$,

$$
\|X\|_{\rho, 2}=\inf \left\{\|z+y\|_{2}: y \in{\overline{\operatorname{Lie}\left(G_{\rho}\right)}}^{\|\|_{2}}\right\},
$$

where $\delta_{\rho}(z)=X$. The infimum is attained in the element $z-\mathbf{P}_{\rho}(z) \in L^{2}\left(\mathcal{M}_{a h}, \tau\right)$, and it belongs to $\mathcal{M}_{a h}$ when $\mathcal{P}$ is orthogonal. Notice that when $\mathcal{P}$ is orthogonal, 
then $\mathbf{P}_{\rho}$ is actually the extension to $L^{2}\left(\mathcal{M}_{a h}, \tau\right)$ of the projection $I-K_{\rho} \circ \delta_{\rho}$. Then, we have

$$
\|X\|_{\rho}=\left\|K_{\rho}(X)\right\|_{2}=\left\|\left(K_{\rho} \circ \delta_{\rho}\right)(z)\right\|_{2}=\left\|z-\mathbf{P}_{\rho}(z)\right\|_{2}=\|X\|_{\rho, 2} .
$$

Thus the quotient metric coincides with the metric given in equation (2.1).

Let us recall the definition of the reductive connection $\nabla^{k}$ introduced in [13]. Suppose that $X, Y$ are tangent fields in $\mathcal{P}$, then the value of $\nabla_{X}^{k} Y$ at $\rho \in \mathcal{P}$ is characterized by the following equation

$$
K_{\rho}\left(\nabla_{X}^{k} Y\right)=X(Y)+\left[K_{\rho}(Y), K_{\rho}(X)\right],
$$

where $X(Y)$ indicates the derivative of $Y$ along $X$. On the other hand, we introduce as in [13] the classifying connection as follows:

$$
K_{\rho}\left(\nabla_{X}^{c} Y\right)=\left(K_{\rho} \circ \delta_{\rho}\right)(X(Y)) .
$$

When $\mathcal{P}$ is orthogonal we shall demonstrate that the mean between $\nabla^{k}$ and $\nabla^{c}$ is the Levi-Civita connection of the metric defined by equation (2.1), and compute its geodesics.

Proposition 2.3. Let $\mathcal{P}$ be an orthogonal homogeneous reductive space. Then the Levi-Civita connection of the metric $\langle,\rangle_{\rho}$ is given by

$$
\nabla=\frac{1}{2}\left(\nabla^{k}+\nabla^{c}\right)
$$

Moreover, $\gamma(t)=L_{e^{t K_{\rho}(X)} \rho}$ is the geodesic adapted to $X \in(T \mathcal{P})_{\rho}$ at $\gamma(0)=\rho$.

Proof. It is easy to show that the reductive connection $\nabla^{k}$ is compatible with the metric. In order to prove that $\nabla^{c}$ is compatible, let us consider $X(t), Y(t)$ two tangent fields along a curve $\alpha(t)$ in $\mathcal{P}$. Since $\mathcal{P}$ is orthogonal, $I-\mathbf{P}_{\rho}$ is an orthogonal projection in $L^{2}\left(\mathcal{M}_{a h}, \tau\right)$ which extends $K_{\rho} \circ \delta_{\rho}$. Then

$$
\left\langle\frac{D^{c} X}{d t}, Y\right\rangle_{\alpha}=\tau\left(K_{\alpha}(Y)^{*}\left(I-\mathbf{P}_{\alpha}\right)\left(\dot{K}_{\alpha}(X)\right)=\tau\left(K_{\alpha}(Y)^{*} \dot{K}_{\alpha}(X)\right) .\right.
$$

We can proceed analogously with the term $\left\langle X, \frac{D^{c} Y}{d t}\right\rangle_{\alpha}$. Then

$$
\begin{aligned}
\frac{d}{d t}\left(\langle X, Y\rangle_{\alpha}\right) & =\frac{d}{d t}\left(\tau\left(K_{\alpha}(Y)^{*} K_{\alpha}(X)\right)\right) \\
& =\tau\left(K_{\alpha}(Y)^{*} \dot{K}_{\alpha}(X)\right)+\tau\left(\dot{K}_{\alpha}(Y)^{*} K_{\alpha}(X)\right) \\
& =\left\langle\frac{D^{c} X}{d t}, Y\right\rangle_{\alpha}+\left\langle X, \frac{D^{c} Y}{d t}\right\rangle_{\alpha} .
\end{aligned}
$$

Thus $\nabla^{c}$ is compatible. In [13] was proved that the connection $\nabla^{c}$ has the same geodesics as $\nabla^{k}$, but with opposite torsion. Therefore $\nabla=\frac{1}{2}\left(\nabla^{k}+\nabla^{c}\right)$ is the Levi-Civita connection. The geodesics of the reductive connection, and hence of our Levi-Civita connection, were also computed in [13]. 
We end this section recalling a useful notion. For any curve $\gamma \in \mathcal{P}$ (not necessarily a geodesic) with $\gamma(0)=\rho$, there is an horizontal lifting $\Gamma$ to the unitary group $U_{\mathcal{M}}$, which is characterized by the following properties:

1. $\Pi_{\rho}(\Gamma)=\gamma$.

2. $\Gamma(0)=1$.

3. $\dot{\Gamma} \in \mathcal{H}_{\gamma} \Gamma$.

Moreover, it is also characterized as the unique solution of the linear differential equation:

$$
\left\{\begin{array}{l}
\dot{\Gamma}=K_{\gamma}(\dot{\gamma}) \Gamma \\
\Gamma(0)=1
\end{array}\right.
$$

\section{Geodesics as minimal curves}

We prove several lemmas in the direction of Theorem 3.8 given at the end of this section. The main idea is to compare the lengths of a curve and its horizontal lifting in different norms, and then use a local convexity result given in [3].

Let us first introduce some notation. The length of a curve $\gamma$ in $\mathcal{P}$ measured with the metric given in (2.1) will be denoted by

$$
L(\gamma)=\int_{0}^{1}\|\dot{\gamma}(t)\|_{\gamma(t)} d t
$$

While the length of a curve $\alpha$ in $U_{\mathcal{M}}$ measured with the 2-norm will be denoted by

$$
L_{2}(\alpha)=\int_{0}^{1}\|\dot{\alpha}(t)\|_{2} d t .
$$

Lemma 3.1. Let $\gamma$ be a piecewise smooth curve in $\mathcal{P}$, and let $\Gamma$ be its horizontal lifting. Then,

$$
L(\gamma)=L_{2}(\Gamma)
$$

Proof. Using the definition of horizontal lifting we have:

$$
\begin{aligned}
L_{2}(\Gamma) & =\int_{0}^{1}\|\dot{\Gamma}(t)\|_{2} d t=\int_{0}^{1}\left\|K_{\gamma(t)}(\dot{\gamma}(t)) \Gamma(t)\right\|_{2} d t \\
& =\int_{0}^{1}\left\|K_{\gamma(t)}(\dot{\gamma}(t))\right\|_{2} d t=\int_{0}^{1}\|\dot{\gamma}(t)\|_{\gamma(t)} d t=L(\gamma) .
\end{aligned}
$$

Now we need to introduce another quotient metric, which is defined in [8] by

$$
\|X\|_{\rho, \infty}=\inf \left\{\|z+y\|: y \in \operatorname{Lie}\left(G_{\rho}\right)\right\},
$$

where $\delta_{\rho}(z)=X$. Note that it is also invariant under the action of $U_{\mathcal{M}}$. The length of a curve $\gamma$ in $\mathcal{P}$ measured with this metric will be indicated by

$$
L_{q, \infty}(\gamma)=\int_{0}^{1}\|\dot{\gamma}(t)\|_{\gamma(t), \infty} d t .
$$


While the length of a curve $\alpha$ in $U_{\mathcal{M}}$ measured with the operator norm will be denoted by

$$
L_{\infty}(\alpha)=\int_{0}^{1}\|\dot{\alpha}(t)\| d t .
$$

The next step is to compare the lengths of a curve and its horizontal lifting with these two metrics. In an orthogonal homogeneous space we have $\mathbf{P}_{\rho}\left(\mathcal{M}_{a h}\right) \subseteq \mathcal{M}_{a h}$ for all $\rho \in \mathcal{P}$, then we can restrict $\mathbf{P}_{\rho}$ to $\mathcal{M}_{a h}$ obtaining an idempotent with range $\operatorname{Lie}\left(G_{\rho}\right)$, that we still denote by $\mathbf{P}_{\rho}$. Moreover, we can define this norm continuous idempotent in any homogeneous reductive space since $\operatorname{Lie}\left(G_{\rho}\right)$ is closed and complemented in the norm topology. Given $\rho \in \mathcal{P}$, set $M:=\left\|I-\mathbf{P}_{\rho}\right\|$. Then, observe that this constant is independent of the choice of $\rho$. Indeed, one computes

$$
\left\|I-\mathbf{P}_{L_{u} \rho}\right\|=\left\|I-A d(u) \circ \mathbf{P}_{\rho} \circ A d\left(u^{*}\right)\right\|=\left\|I-\mathbf{P}_{\rho}\right\| .
$$

Lemma 3.2. Let $\gamma$ be a piecewise smooth curve in $\mathcal{P}$, and let $\Gamma$ be its horizontal lifting. Then,

$$
L_{\infty}(\Gamma) \leq M L_{q, \infty}(\gamma) .
$$

Proof. Fix $\rho \in \mathcal{P}$. For $z \in \mathcal{H}_{\rho}$ such that $\delta_{\rho}(z)=X$ and $y \in \operatorname{Lie}\left(G_{\rho}\right)$, we have

$$
\|z\|=\left\|\left(I-\mathbf{P}_{\rho}\right)(z)\right\| \leq M\|z+y\| .
$$

Then,

Therefore,

$$
\|z\| \leq M\left\|\delta_{\rho}(z)\right\|_{\rho, \infty}
$$

$$
\left\|K_{\rho}(X)\right\| \leq M\left\|\delta_{\rho}\left(K_{\rho}(X)\right)\right\|_{\rho, \infty}=M\|X\|_{\rho, \infty} .
$$

Using this inequality, and the fact previously noted that $M$ is independent of the point, one finally obtains

$$
\begin{aligned}
L_{\infty}(\Gamma) & =\int_{0}^{1}\|\dot{\Gamma}(t)\| d t=\int_{0}^{1}\left\|K_{\gamma(t)}(\dot{\gamma}(t)) \Gamma(t)\right\| d t \\
& =\int_{0}^{1}\left\|K_{\gamma(t)}(\dot{\gamma}(t))\right\| d t \leq M \int_{0}^{1}\|\dot{\gamma}(t)\|_{\gamma(t), \infty}=M L_{q, \infty}(\gamma) .
\end{aligned}
$$

We need some facts about the geometry of the unitary group $U_{\mathcal{M}}$. The curves $\delta(t)=u e^{t z}$, where $u \in \mathcal{M}$ and $z \in \mathcal{M}_{a h}$ such that $\|z\|<\pi$, have minimal length along their paths when one measures lengths with the $p$-norm, $p \geq 2$ (see [1]). Based on this fact, in Theorem 2.1 of [3] it was proved that if $F_{2}$ denotes the energy functional

$$
F_{2}(\alpha)=\int_{0}^{1}\|\dot{\alpha}\|_{2}^{2} d t=\int_{0}^{1} \tau\left(\dot{\alpha}^{*} \dot{\alpha}\right) d t,
$$

where $\alpha$ is a piecewise smooth curve in $U_{\mathcal{M}}$ and $\alpha_{s}(t)$ is a smooth variation of $\alpha$, i.e.

$$
\alpha_{s}(t) \in U_{\mathcal{M}}, \quad s \in(-r, r), \quad t \in[0,1], \quad \alpha_{0}=\alpha,
$$


then the first variation of the energy functional is given by

$$
\left.\frac{1}{2} \frac{d}{d s} F_{2}\left(\alpha_{s}\right)\right|_{s=0}=\left.\tau\left(x_{0} y_{0}\right)\right|_{t=0} ^{t=1}-\int_{0}^{1} \tau\left(\frac{d}{d t}\left[x_{0}\right] y_{0}\right) d t,
$$

where $x_{s}(t)=\alpha_{s}(t)^{*} \frac{d}{d t} \alpha_{s}(t)$ and $y_{s}(t)=\alpha_{s}(t)^{*} \frac{d}{d s} \alpha_{s}(t)$.

Our result relies in the main theorem of [3].

Proposition 3.3. (Theorem 4.5 of [3]) Let $u_{0}, u_{1}, u_{2} \in U_{\mathcal{M}}$, such that $\left\|u_{i}-u_{j}\right\|<$ $\frac{\sqrt{2-\sqrt{2}}}{2}$. Let $\delta(t)=u_{1} e^{t z}$ be the minimal geodesic joining $u_{1}$ and $u_{2}$. Then $f(s)=$ $d_{p}\left(u_{0}, \delta(s)\right)^{p}, s \in[0,1]$ ( $d_{p}=$ geodesic distance induced by the $p$-norm) is a convex function, for $p$ an even integer.

We shall use this result when $p=2$.

Remark 3.4. If we compute the differential at $0 \in \mathcal{M}_{a h}$ of the exponential map at $\rho$, i.e.

we obtain

$$
\exp _{\rho}: \mathcal{H}_{\rho} \longrightarrow \mathcal{P}, \quad \exp _{\rho}(z)=L_{e^{z}} \rho
$$

$$
\left(d \exp _{\rho}\right)_{0}: \mathcal{H}_{\rho} \longrightarrow(T \mathcal{P})_{\rho}, \quad\left(d \exp _{\rho}\right)_{0}(z)=\delta_{\rho}(z)
$$

which is an isomorphism. Therefore by the inverse function theorem there exists $r>0$ such that in a ball $B_{r}(0)$ we have that

$$
\exp _{\rho}: B_{r}(0) \longrightarrow \exp _{\rho}\left(B_{r}(0)\right)
$$

is a diffeomorphism. Let us write $\mathcal{V}_{r}(\rho)=\exp _{\rho}\left(B_{r}(0)\right)$ for short. Then, for each $\rho_{1} \in \mathcal{V}_{r}(\rho)$, there exists a unique geodesic given by $\exp _{\rho}(t z)=L_{e^{t z}} \rho$ joining $\rho$ and $\rho_{1}$ inside $\mathcal{V}_{r}(\rho)$, where $z$ satisfies $L_{e^{z}} \rho=\rho_{1}$.

Remark 3.5. Now consider the following map

$$
F: \mathcal{H}_{\rho} \oplus \operatorname{Lie}\left(G_{\rho}\right) \longrightarrow U_{\mathcal{M}}, \quad F((z, x))=e^{z} e^{x}
$$

Differentiating one obtains

$$
(d F)_{(0,0)}: \mathcal{M}_{a h} \longrightarrow \mathcal{M}_{a h}, \quad(d F)_{(0,0)}(z, x)=z+x,
$$

which is an isomorphism. Then there exists a neighborhood $V$ of $(0,0)$ and an $\epsilon>0$ such that

$$
F: V \longrightarrow B_{\epsilon}(1) \cap U_{\mathcal{M}}
$$

is a diffeomorphism. Moreover, we can choose $V \subseteq B_{\epsilon_{1}}(0) \times B_{\epsilon_{2}}(0)$ for $\epsilon_{1}, \epsilon_{2}$ so small as we want if we just adjust $\epsilon$. In the remainder of this section we require $\epsilon$, $\epsilon_{1}, \epsilon_{2}$ to satisfy:
i) $\epsilon<\frac{\sqrt{2-\sqrt{2}}}{2}$.
ii) $\epsilon_{i}<\frac{\pi}{9}$, for $i=1,2$.
iii) $\epsilon_{1}<r$. 
Lemma 3.6. Let $\gamma$ be a piecewise smooth curve in $\mathcal{P}$ such that $\gamma(0)=\rho$ and $L_{q, \infty}(\gamma)<\epsilon / M$. Then there exists $z \in \mathcal{H}_{\rho}$ satisfying:

i) $\Gamma(1)=e^{z} e^{x}$, where $x \in \operatorname{Lie}\left(G_{\rho}\right)$.

ii) $z \in B_{r}(0)$ is unique such that $L_{e^{z}} \rho=\gamma(1)$.

iii) The elements $1, e^{z}$ and $\Gamma(1)$ lie closer than $\frac{\sqrt{2-\sqrt{2}}}{2}$ in norm.

Proof. i) If we write $d_{\infty}$ for the geodesic distance in $U_{\mathcal{M}}$ with the Finsler metric given by the operator norm, then using the Lemma 3.2 we obtain

$$
\|1-\Gamma(1)\| \leq d_{\infty}(1, \Gamma(1)) \leq L_{\infty}(\Gamma) \leq M L_{q, \infty}(\gamma)<\epsilon .
$$

By Remark 3.5 there exists a unique $(z, x) \in \mathcal{H}_{\rho} \oplus \operatorname{Lie}\left(G_{\rho}\right)$ such that $\|z\|<\epsilon_{1}$, $\|x\|<\epsilon_{2}$ and $e^{z} e^{x}=\Gamma(1)$.

ii) Note that $L_{e^{z}} \rho=L_{e^{z} e^{x}} \rho=L_{\Gamma(1)} \rho=\gamma(1)$. Moreover, it is unique because $\|z\|<\epsilon_{1}<r$ and $\exp _{\rho}$ is one to one on the ball of radius $r$.

iii) We just have shown $\|1-\Gamma(1)\|<\epsilon<\frac{\sqrt{2-\sqrt{2}}}{2}$. On the other hand, our choice of $\epsilon_{2}$ implies

$$
\left\|e^{z}-\Gamma(1)\right\|=\left\|1-e^{x}\right\|=\sqrt{2-2 \cos (\|x\|)}<\frac{\sqrt{2-\sqrt{2}}}{2} .
$$

Analogously, our choice of $\epsilon_{1}$ gives

$$
\left\|1-e^{z}\right\|=\sqrt{2-2 \cos (\|z\|)}<\frac{\sqrt{2-\sqrt{2}}}{2} .
$$

Proposition 3.7. Let $\mathcal{P}$ be an orthogonal homogeneous reductive space. Consider $\gamma$ a piecewise smooth curve in $\mathcal{P}$ such that $\gamma(0)=\rho$ and $L_{q, \infty}(\gamma)<\epsilon / M$. Then there exists a geodesic $\delta(t)=L_{e^{t z}} \rho$ satisfying $\delta(1)=\gamma(1)$ and $L(\delta) \leq L(\gamma)$.

Proof. In view of Lemma 3.6 we have $\gamma(1)=L_{e^{z}} \rho$, where $\Gamma(1)=e^{z} e^{x}, e^{z}$ and 1 lie at distance less than $\frac{\sqrt{2-\sqrt{2}}}{2}$ in norm. Consider now $\mu(s)=e^{z} e^{s x}, s \in[0,1]$, the minimal geodesic joining $e^{z}$ and $\Gamma(1)=e^{z} e^{x}$. Therefore by Proposition 3.3 the function $f(s)=d_{2}(1, \mu(s))^{2}$ is convex.

Claim: $f^{\prime}(0)=0$.

Note the following:

$$
\begin{aligned}
\|\mu(s)-1\| & \leq\left\|e^{z} e^{s x}-e^{s x}\right\|+\left\|e^{s x}-1\right\| \leq\left\|e^{z}-1\right\|+\left\|e^{x}-1\right\| \\
& =\left\|e^{z}-1\right\|+\left\|\Gamma(1)-e^{z}\right\|<\sqrt{2-\sqrt{2}}<2 .
\end{aligned}
$$

Then the antihermitic logarithm

$$
\log :\left\{u \in U_{M}:\|u-1\|<2\right\} \longrightarrow\left\{y \in M_{a h}:\|y\|<\pi\right\}
$$

is well defined and smooth. Let us call $\epsilon_{s}(t)=e^{t \log (\mu(s))}$, which is a smooth variation of $\epsilon_{0}(t)=e^{t z}$. For each $s$, these curves are minimal geodesics in $U_{\mathcal{M}}$ as a consequence of the inequality $\|\log (\mu(s))\|<\pi$. Therefore,

$$
f(s)=L_{2}\left(\epsilon_{s}\right)^{2}=\|\log (\mu(s))\|_{2}^{2}=F_{2}\left(\epsilon_{s}\right) .
$$


Following the notation adopted in equation (3.1) one notes that $x_{s}(t)=\log (\mu(s))$ is constant with respect to $t$. Thus, the derivative reduces to

$$
f^{\prime}(0)=2\left(\tau\left(z y_{0}(1)\right)-\tau\left(z y_{0}(0)\right)\right) .
$$

Then observe that $y_{0}(0)=\left.\epsilon_{0}(0)^{*} \frac{d}{d s}\right|_{s=0} \epsilon_{s}(0)=0$. On the other hand, we have to compute $y_{0}(1)=\left.\epsilon_{0}(1)^{*} \frac{d}{d s}\right|_{s=0} \epsilon_{s}(1)=x$, and using that $\mathcal{H}_{\rho}$ and $\operatorname{Lie}\left(G_{\rho}\right)$ are $\tau$ orthogonal, we finally obtain

$$
\tau\left(z y_{0}(1)\right)=\tau(z x)=0 .
$$

Thus our claim is proved. From this fact and the convexity of $f$ we deduce that it has a global minimum at $s=0$. Therefore,

$$
L_{2}\left(\epsilon_{0}\right)=d_{2}\left(1, e^{z}\right)=f(0)^{1 / 2} \leq f(1)^{1 / 2}=d_{2}(1, \Gamma(1)) \leq L_{2}(\Gamma) .
$$

Calling $\delta$ the unique geodesic with the same initial and final endpoints as $\gamma$, note that $\epsilon_{0}$ is the horizontal lifting of $\delta$. Then by Lemma 3.1 we can conclude

$$
L(\delta)=L_{2}\left(\epsilon_{0}\right) \leq L_{2}(\Gamma)=L(\gamma) .
$$

We call a piecewise smooth curve a geodesic polygon if it is a continuous path in $\mathcal{P}$, and consists of geodesics paths glued together. As an immediate consequence of Proposition 3.7 we give the following result.

Corollary. Let $\mathcal{P}$ be an orthogonal homogeneous reductive space and $\gamma$ a piecewise smooth curve in $\mathcal{P}$. Then there exists a geodesic polygon $\nu$ such that $\nu(0)=\gamma(0)$, $\nu(1)=\gamma(1)$ and

$$
L(\nu) \leq L(\gamma)
$$

Proof. Clearly we can suppose that $\gamma$ is smooth. Consider a partition $0=t_{0}<$ $t_{1}<\ldots<t_{n}=1$ such that $L_{q, \infty}\left(\left.\gamma\right|_{\left[t_{i}, t_{i+1}\right]}\right)<\epsilon / M$. We use Proposition 3.7 to find geodesics $\delta_{i}$ with the same endpoints as $\left.\gamma\right|_{\left[t_{i}, t_{i+1}\right]}$ for $i=0, \ldots, n-1$ satisfying

$$
L\left(\delta_{i}\right) \leq L\left(\left.\gamma\right|_{\left[t_{i}, t_{i+1}\right]}\right) \text {. }
$$

Then the curve $\nu$ obtained by gluing the geodesics $\delta_{i}$ is a geodesic polygon shorter than $\gamma$.

In the next result we shall take $R:=\min \{r, \epsilon / 2 M\}$.

Theorem 3.8. Let $\mathcal{P}$ be an orthogonal homogeneous reductive space and $\rho \in \mathcal{P}$. Given any $\rho_{1} \in \mathcal{V}_{R}(\rho)$, there exists a unique geodesic inside $\mathcal{V}_{R}(\rho)$ which has minimal length among all the piecewise smooth curves inside $\mathcal{V}_{R}(\rho)$, joining the points $\rho$ and $\rho_{1}$.

Proof. Let $\gamma$ be a piecewise smooth curve inside $\mathcal{V}_{R}(\rho)$. We can take a partition of the unit interval $0=t_{0}<t_{1}<\ldots<t_{n}=1$ such that $L_{q, \infty}\left(\left.\gamma\right|_{\left[t_{i}, t_{i+1}\right]}\right)<\epsilon / 2 M$. Therefore,

$$
L_{q, \infty}\left(\left.\gamma\right|_{\left[t_{0}, t_{2}\right]}\right)=L_{q, \infty}\left(\left.\gamma\right|_{\left[t_{0}, t_{1}\right]}\right)+L_{q, \infty}\left(\left.\gamma\right|_{\left[t_{1}, t_{2}\right]}\right)<\epsilon / 2 M+\epsilon / 2 M=\epsilon / M .
$$


By Lemma 3.6 there is a unique $z_{2} \in B_{r}(0) \subseteq \mathcal{H}_{\rho}$ such that $L_{e^{z_{2}}} \rho=\gamma\left(t_{2}\right)$. Applying Proposition 3.7, the geodesic $\delta_{2}(t)=L_{e^{t z_{2}}} \rho$ satisfies $L\left(\delta_{2}\right) \leq L\left(\left.\gamma\right|_{\left[t_{0}, t_{2}\right]}\right)$. On the other hand, since we have $\gamma\left(t_{2}\right) \in \mathcal{V}_{R}(\rho)$, by Remark 3.4 there exists a unique $\overline{z_{2}} \in B_{R}(0)$ such that $L_{e^{\overline{z_{2}}}} \rho=\gamma\left(t_{2}\right)$. Using our assumption that $R \leq r$, we have $z_{2}=\overline{z_{2}}$, and then $\delta_{2}$ is the unique geodesic in $\mathcal{V}_{R}(\rho)$ joining $\rho$ and $\gamma\left(t_{2}\right)$. An easy computation shows that $L_{q, \infty}\left(\delta_{2}\right)=\left\|z_{2}\right\|$. Therefore, if we use the symbol \# to denote a path obtained by adjoining two consecutive paths, we get

$$
L_{q, \infty}\left(\left.\delta_{2} \# \gamma\right|_{\left[t_{2}, t_{3}\right]}\right)=L_{q, \infty}\left(\delta_{2}\right)+L_{q, \infty}\left(\left.\gamma\right|_{\left[t_{2}, t_{3}\right]}\right)<\left\|z_{2}\right\|+\epsilon / 2 M<\epsilon / M .
$$

Thus by the same argument as before, there exists a unique geodesic $\delta_{3}$ in $\mathcal{V}_{R}(\rho)$ such that $L\left(\delta_{3}\right) \leq L\left(\left.\delta_{2} \# \gamma\right|_{\left[t_{2}, t_{3}\right]}\right)$. Moreover, we can also estimate

$$
\begin{aligned}
L\left(\delta_{3}\right) & \leq L\left(\left.\delta_{2} \# \gamma\right|_{\left[t_{2}, t_{3}\right]}\right)=L\left(\delta_{2}\right)+L\left(\left.\gamma\right|_{\left[t_{2}, t_{3}\right]}\right) \\
& \leq L\left(\left.\gamma\right|_{\left[t_{0}, t_{2}\right]}\right)+L\left(\left.\gamma\right|_{\left[t_{2}, t_{3}\right]}\right)=L\left(\left.\gamma\right|_{\left[t_{0}, t_{3}\right]}\right) .
\end{aligned}
$$

It is clear that we can finish the proof by an inductive argument.

Remark 3.9. Our choice of $R>0$ works for any $\rho \in \mathcal{P}$. To show this, first observe that $R$ depends only on $r, M$ and $\epsilon$.

1. $r$ is independent of the point because the action is isometric.

2. The independence of $M$ was shown before the Lemma 3.2.

3. If we considerer the map $F^{u}: \mathcal{H}_{L_{u} \rho} \oplus \operatorname{Lie}\left(G_{L_{u} \rho}\right) \longrightarrow U_{M}$, it is straightforward to verify that $F^{u}=F \circ A d(u)$. Then, using that $A d(u)$ is an isometric isomorphism we obtain that $F^{u}$ is a local diffeomorphism if and only if $F$ is also a local diffeomorphism.

Remark 3.10. All the work of this section could be carried out in $C^{*}$-algebra with a faithful trace. However, as we shall see, in the following examples we need to work in a finite von Neumann algebra to prove the orthogonality condition.

\section{Examples}

We give several examples of orthogonal homogeneous reductive spaces. In these examples, the fundamental step to ensure that Theorem 3.8 holds, consists in proving the orthogonal condition. The following situation frequently arises: we have an algebraic subgroup $G$ (in the sense of [6], [11]) of the Banach-Lie group $U_{\mathcal{M}}$ and we want to check that $G$ is actually a Banach-Lie subgroup to give a smooth manifold structure in the quotient $U_{\mathcal{M}} / G$. In view of Theorem 4.18 of [6] we only have to prove that $\operatorname{Lie}(G)$ is complemented in $\mathcal{M}_{a h}$. On the other hand, to obtain the minimality of geodesics, we must prove the orthogonality condition. So we can obtain both properties if we check that the orthogonal projection

$$
\mathbf{P}: L^{2}\left(\mathcal{M}_{a h}, \tau\right) \longrightarrow \overline{\operatorname{Lie}(G)} \|^{\|}
$$


satisfies $\mathbf{P}\left(\mathcal{M}_{a h}\right) \subseteq \operatorname{Lie}(G)$. Moreover, as we observe in Remark 2.1, this gives invariant supplements under the inner action of $U_{\mathcal{M}}$, and therefore, a reductive structure in $U_{\mathcal{M}} / G$.

\subsection{Unitary orbit of a state}

This first example is concerned with the unitary orbit of a state. Let $\mathcal{S}_{\mathcal{M}}$ be the set of normal faithful states of $\mathcal{M}$. Consider the action

$$
L: U_{\mathcal{M}} \times \mathcal{S}_{\mathcal{M}} \longrightarrow \mathcal{S}_{\mathcal{M}}
$$

given by

$$
L_{u} \varphi=\varphi \circ A d\left(u^{*}\right), \quad u \in U_{\mathcal{M}}, \varphi \in \mathcal{S}_{\mathcal{M}} .
$$

For $\varphi \in \mathcal{S}_{\mathcal{M}}$, we denote by $\mathcal{U}_{\varphi}$ the unitary orbit of $\varphi$, i.e.

$$
\mathcal{U}_{\varphi}=\left\{\varphi \circ A d\left(u^{*}\right): u \in U_{M}\right\} .
$$

The isotropy group under this action

$$
G_{\varphi}=\left\{u \in U_{\mathcal{M}}: \varphi(u y)=\varphi(y u), \forall y \in \mathcal{M}\right\}
$$

is an algebraic subgroup of order $\leq 1$ of the Lie-Banach group $U_{\mathcal{M}}$. Actually this is verified by taking the polynomials

$$
p_{y}: \mathcal{M} \times \mathcal{M} \longrightarrow \mathbb{C}, p_{y}((a, b))=\varphi(a y)-\varphi(y a), \quad y \in \mathcal{M} .
$$

The Lie algebra

$$
\operatorname{Lie}\left(G_{\varphi}\right)=\left\{x \in \mathcal{M}_{a h}: \varphi(x y)=\varphi(y x), \forall y \in \mathcal{M}\right\}
$$

consists in the antihermitic elements of the centralizer of $\varphi$, which is a von Neumann subalgebra of $\mathcal{M}$. It is a well known fact that in a finite algebra there exists a unique trace invariant conditional expectation onto any von Neumann subalgebra (see for instance [16]). This gives that $\operatorname{Lie}\left(G_{\varphi}\right)$ is complemented in $\mathcal{M}_{a h}$, and therefore, the set $U_{\mathcal{M}} / G_{\varphi}$ has a smooth manifold structure. Then we endow $\mathcal{U}_{\varphi}$ with the manifold structure such that the bijection $[u] \mapsto \varphi \circ \operatorname{Ad}\left(u^{*}\right)$ is a diffeomorphism.

Observe that the hypothesis of Theorem 3.8 is verified because this conditional expectation extends to the orthogonal projection over the respective completions with the 2 -norm. Thus, we can conclude that the curve $\gamma(t)=\varphi \circ A d\left(e^{-t z} u^{*}\right)$ has minimal length among all the curves contained in a neighborhood of $\varphi \circ A d\left(u^{*}\right)$ that start at this point.

\subsection{Unitary orbit of a normal element}

Let $a$ be a normal element of $\mathcal{M}$. We can study the unitary orbit of $a$, that is the set

$$
\mathcal{U}(a)=\left\{u a u^{*}: u \in U_{\mathcal{M}}\right\} .
$$

The isotropy group at $a$ of the natural action of $U_{\mathcal{M}}$ is given by

$$
G_{a}=\left\{u \in U_{\mathcal{M}}: u a=a u\right\} .
$$

Clearly, it is an algebraic subgroup of $U_{\mathcal{M}}$ of order $\leq 1$. The polynomials are defined by $p: \mathcal{M} \times \mathcal{M} \longrightarrow \mathcal{M}, p((c, d))=c a-a c$. As in the previously example, 
the Lie algebra of the isotropy group is the antihermitic elements of a von Neumann subalgebra

$$
\operatorname{Lie}\left(G_{a}\right)=\left\{x \in M_{a h}: x a=a x,\right\}=\{a\}^{\prime} \cap \mathcal{M}_{a h} .
$$

Thus $\mathcal{U}(a) \cong U_{\mathcal{M}} / G_{a}$ is a smooth manifold, and the geodesics $\gamma(t)=e^{t z} a e^{-t z}$ are locally minimizing.

\subsection{Unitary orbit of a spectral measure}

Let $\Sigma$ be a $\sigma$-algebra of subsets of some set. Let $E: \Sigma \longrightarrow \mathcal{M}$ be a spectral measure whose values are selfadjoint projections in $\mathcal{M}$. The unitary orbit of $E$ is

$$
\mathcal{U}(E)=\left\{u E(.) u^{*}: u \in U_{\mathcal{M}}\right\}
$$

We take as subgroup of order $\leq 1$ the isotropy group under the natural action of $U_{\mathcal{M}}$ as in the preceding examples, i.e.

$$
G_{E}=\left\{u \in U_{\mathcal{M}}: u E(S)=E(S) u, \quad \forall S \in \Sigma\right\} .
$$

The polynomials are

$$
p_{S}: \mathcal{M} \times \mathcal{M} \longrightarrow \mathcal{M}, p_{S}((a, b))=a E(S)-E(S) a .
$$

The Lie algebra of this group is

$$
\operatorname{Lie}\left(G_{E}\right)=\left\{x \in \mathcal{M}_{a h}: x E(S)=E(S) x, \forall S \in \Sigma\right\},
$$

which consists of the antihermitic elements of a von Neumann subalgebra. Then we can use Theorem 3.8 to show that in the orthogonal homogeneous reductive space $\mathcal{U}(E) \cong U_{\mathcal{M}} / G_{E}$ the geodesics $\gamma(t)=e^{t z} E(.) e^{-t z}$ are locally minimizing.

\subsection{Unitary orbit of a $*$-homomorphism}

Consider $\psi: \mathcal{M} \longrightarrow \mathcal{M}$ a $*$-homomorphism. Again we take the unitary orbit of $\psi$, i.e.

$$
\mathcal{U}(\psi)=\left\{u \psi(.) u^{*}: u \in U_{\mathcal{M}}\right\} .
$$

The algebraic subgroup of order $\leq 1$ is the isotropy at $\psi$ under the natural action of the unitary group

$$
G_{\psi}=\left\{u \in U_{M}: u \psi(y)=\psi(y) u, \forall y \in M\right\} .
$$

The polynomials used to prove this fact are

$$
p_{y}: \mathcal{M} \times \mathcal{M} \longrightarrow \mathcal{M}, p_{y}((a, b))=a \psi(y)-\psi(y) a .
$$

The Lie algebra consists in the antihermitic elements of the von Neumann algebra given by the commutant of $\psi(\mathcal{M})$. Therefore, $\mathcal{U}(\psi) \cong U_{\mathcal{M}} / G_{\psi}$ is an orthogonal homogeneous reductive space where the Theorem 3.8 holds. 


\subsection{Partial isometries}

This example is concerned with partial isometries in $\mathcal{M}$. The set of partial isometries is

$$
\mathcal{I}=\left\{v \in \mathcal{M}: v^{*} v \text { is a projection }\right\} .
$$

We can give an action of the unitary group $U_{\mathcal{M}} \times U_{\mathcal{M}}$ of $\mathcal{M} \times \mathcal{M}$ on $\mathcal{I}$ by acting both on the initial and the final spaces. The action is given by

$$
L:\left(U_{\mathcal{M}} \times U_{\mathcal{M}}\right) \times \mathcal{I} \longrightarrow \mathcal{I}, \quad L_{(u, w)} v=u v w^{*} .
$$

This action is locally transitive, i.e. if two partial isometries lie closer than $1 / 2$ in the operator norm, then they are conjugate by a pair of unitaries. In [2] was proved that each connected component, which as a consequence of local transitivity coincides with an orbit, is a homogeneous space of $U_{\mathcal{M}} \times U_{\mathcal{M}}$ and a $C^{\infty}$ submanifold of $\mathcal{M}$. Hence, by Remark 2.1 , to give a reductive structure we just have to prove the orthogonality condition.

Fix $v \in \mathcal{I}$, we shall study its orbit $\mathcal{O}(v)$. The isotropy group at $v$ is

$$
G_{v}=\left\{(u, w) \in U_{\mathcal{M}} \times U_{\mathcal{M}}: u v=v w\right\} .
$$

Note that if $(u, w) \in G_{v}$, then $u$ commutes with the final projection $v v^{*}$ and $w$ commutes with the initial projection $v^{*} v$. One can compute the Lie algebra of this group

$$
\begin{aligned}
\operatorname{Lie}\left(G_{v}\right) & =\left\{(x, y) \in \mathcal{M}_{a h} \times \mathcal{M}_{a h}: x v=v y\right\} \\
& =\left\{\left(\left(\begin{array}{cc}
x_{11} & 0 \\
0 & x_{22}
\end{array}\right),\left(\begin{array}{cc}
v^{*} x_{11} v & 0 \\
0 & y_{22}
\end{array}\right)\right): x_{11}, x_{22}, y_{22} \text { antihermitic }\right\},
\end{aligned}
$$

where the matrix decomposition is respect to $v v^{*}$ in the first coordinate and to $v^{*} v$ in the second coordinate. Note that in this case the Lie algebra does not consist of the antihermitic elements of a von Neumann algebra. In a finite von Neumann algebra, the orbits have the following particular property.

Claim: Let $\mathcal{M}$ be a finite von Neumann algebra, then $\mathcal{O}(v)=\mathcal{O}\left(v^{*} v\right)$. In particular, there is a projection in each orbit.

To prove our claim, consider the set of partial isometries with fixed initial space. In other words, if $p$ is a projection, we look at the set

$$
\mathcal{I}_{p}=\left\{v \in \mathcal{M}: v^{*} v=p\right\} .
$$

First let us demonstrate that $\left\{u p: u \in U_{\mathcal{M}}\right\}=\mathcal{I}_{p}$. One inclusion is trivial, for the other take $v \in \mathcal{I}_{p}$, and let $q=v v^{*}$, which is a projection that is equivalent with $p$. Since $\mathcal{M}$ is finite, there exists $u \in U_{\mathcal{M}}$ such that $u q u^{*}=p$. Note that the element $1-p+u v$ is unitary, and therefore, the element $w=u^{*}(1-p)+v$ is also unitary. Finally, we obtain $w p=v p=v$.

Then our claim follows easily. Indeed, since $v \in \mathcal{I}_{v^{*} v}=\left\{u v^{*} v: u \in U_{\mathcal{M}}\right\}$, there is $u \in U_{\mathcal{M}}$ such that $v=u\left(v^{*} v\right)=u\left(v^{*} v\right) 1$. Thus, we obtain $\mathcal{O}(v)=\mathcal{O}\left(v^{*} v\right)$. 
As a corollary of the above claim, it suffices to study the isotropy at a projection $p$. In this case, the expression of the Lie algebra reduces to

$$
\operatorname{Lie}\left(G_{p}\right)=\left\{\left(\left(\begin{array}{cc}
x_{11} & 0 \\
0 & x_{22}
\end{array}\right),\left(\begin{array}{cc}
x_{11} & 0 \\
0 & y_{22}
\end{array}\right)\right): x_{11}, x_{22}, y_{22} \text { antihermitic }\right\},
$$

where the matrix decompositions are both respect to the projection $p$. Let us define a real bounded projection onto $\operatorname{Lie}\left(G_{p}\right)$ by

$\mathbf{P}: \mathcal{M}_{a h} \times \mathcal{M}_{a h} \longrightarrow \operatorname{Lie}\left(G_{p}\right)$,

$$
\left(\left(\begin{array}{cc}
x_{11} & x_{12} \\
-x_{12}^{*} & x_{22}
\end{array}\right),\left(\begin{array}{cc}
y_{11} & y_{12} \\
-y_{12}^{*} & y_{22}
\end{array}\right)\right) \mapsto\left(\left(\begin{array}{cc}
\frac{x_{11}+y_{11}}{2} & 0 \\
0 & x_{22}
\end{array}\right),\left(\begin{array}{cc}
\frac{x_{11}+y_{11}}{2} & 0 \\
0 & y_{22}
\end{array}\right)\right)
$$

Observe that the kernel of this projection is

$$
\operatorname{ker}(\mathbf{P})=\left\{\left(\left(\begin{array}{cc}
w & c_{12} \\
-c_{12}^{*} & 0
\end{array}\right),\left(\begin{array}{cc}
-w & d_{12} \\
-d_{12}^{*} & 0
\end{array}\right)\right): w, c_{12}, d_{12} \text { antihermitic }\right\} .
$$

Using the trace $\tau$ of $\mathcal{M}$, we can define a finite trace in $\mathcal{M} \times \mathcal{M}$ by

$$
\tilde{\tau}((x, y))=\frac{\tau(x)+\tau(y)}{2}, \quad x, y \in \mathcal{M} .
$$

This gives an inner product on $\mathcal{M} \times \mathcal{M}$ such that $\operatorname{ker}(\mathbf{P})$ is orthogonal to $\operatorname{Lie}\left(G_{p}\right)$. Indeed, for $(x, y) \in \operatorname{Lie}\left(G_{p}\right),(c, d) \in \operatorname{ker}(\mathbf{P})$, one obtains

$$
\begin{aligned}
2 \tilde{\tau}((x, y)(c, d))= & -\tau(x c)-\tau(y d) \\
= & -\tau\left(\left(\begin{array}{cc}
x_{11} & 0 \\
0 & x_{22}
\end{array}\right)\left(\begin{array}{cc}
w & c_{12} \\
-c_{12}^{*} & 0
\end{array}\right)\right)- \\
& -\tau\left(\left(\begin{array}{cc}
x_{11} & 0 \\
0 & y_{22}
\end{array}\right)\left(\begin{array}{cc}
-w & d_{12} \\
-d_{12}^{*} & 0
\end{array}\right)\right) \\
= & -\tau\left(\left(\begin{array}{cc}
x_{11} w & 0 \\
0 & 0
\end{array}\right)\right)+\tau\left(\left(\begin{array}{cc}
x_{11} w & 0 \\
0 & 0
\end{array}\right)\right)=0 .
\end{aligned}
$$

By the orthogonality of $\operatorname{ker}(\mathbf{P})$ with its range, $\mathbf{P}$ extends to the real orthogonal projection onto $\overline{\operatorname{Lie}\left(G_{p}\right)} \|^{\|}$. Thus, we obtain that $\mathcal{O}(v)=\mathcal{O}\left(v^{*} v\right)$ is an orthogonal homogeneous reductive space. Therefore the geodesics $\gamma(t)=e^{t z_{1}} u v w^{*} e^{-t z_{2}}$ have minimal length among all the curves inside a neighborhood of $u v w^{*}$.

\subsection{Unitary orbit of a conditional expectation}

Let $\mathcal{N}$ be a von Neumann subalgebra of $\mathcal{M}$ and $E: \mathcal{M} \longrightarrow \mathcal{N}$ the unique trace invariant conditional expectation. Our next example is about the unitary orbit of $E$. For a treatment of geometric properties of this example in a more general setting than finite algebras, we refer the reader to [4] and [5].

Let us define an action of $U_{\mathcal{M}}$ on the algebra $\mathcal{B}(\mathcal{M})$ of bounded operators on $\mathcal{M}$ by

$$
L: U_{\mathcal{M}} \times \mathcal{B}(\mathcal{M}) \longrightarrow \mathcal{B}(\mathcal{M}), \quad L_{u} T=A d(u) \circ T \circ A d\left(u^{*}\right) .
$$


Consider the unitary orbit of $E$ with this action

$$
\mathcal{U}(E)=\left\{L_{u} E: u \in \mathcal{M}\right\} .
$$

The isotropy group at $E$ is usually called the normalizer of $E$,

$$
\mathcal{N}_{E}=\left\{u \in U_{\mathcal{M}}: E\left(u y u^{*}\right)=u E(y) u^{*}, y \in \mathcal{M}\right\} .
$$

Let us show that $\mathcal{N}_{E}$ is an algebraic subgroup of $U_{\mathcal{M}}$ of order $\leq 2$. For each $y \in \mathcal{M}$ define the following bilinear maps

$$
\psi_{y}:(\mathcal{M} \times \mathcal{M}) \times(\mathcal{M} \times \mathcal{M}) \longrightarrow \mathcal{M}, \quad \psi_{y}\left((a, b),\left(a^{\prime}, b^{\prime}\right)\right)=E\left(a y b^{\prime}\right)-a E(y) b^{\prime} .
$$

Then take the polynomials

$$
p_{y}((a, b))=\psi_{y}((a, b),(a, b))=E(a y b)-a E(y) b .
$$

In [5] was proved that for any faithful normal conditional expectation, its unitary orbit is a homogeneous reductive space of $U_{\mathcal{M}}$. In the finite algebra case we shall prove the orthogonality condition restricting to the unique trace invariant conditional expectation $E$. The arguments involved are adapted from Proposition 4.5 in [5], to this easier case.

The Lie algebra of $\mathcal{N}_{E}$ is the kernel of the differential of the natural fibration

$$
\Pi_{E}: U_{\mathcal{M}} \longrightarrow \mathcal{U}(E), \quad \Pi_{E}(u)=L_{u} E .
$$

In [5] was pointed out that

$$
\operatorname{Lie}\left(\mathcal{N}_{E}\right)=\operatorname{ker}\left(\left(d \Pi_{E}\right)_{1}\right)=\left(\mathcal{N}+\mathcal{M}_{E}\right) \cap \mathcal{M}_{a h},
$$

where $\mathcal{M}_{E}$ is the von Neumann subalgebra of $\mathcal{M}$ given by

$$
\mathcal{M}_{E}=\left\{x \in \mathcal{N}^{\prime} \cap \mathcal{M}: E(x y)=E(y x), \forall y \in \mathcal{M}\right\} .
$$

Let us call $F: \mathcal{M} \longrightarrow \mathcal{M}_{E}$ the unique conditional expectation such that $\tau \circ F=\tau$. Denote by $Z(\mathcal{M})$ the center of $\mathcal{M}$. Note that $E\left(\mathcal{M}_{E}\right)=Z(\mathcal{N})$, then we have a conditional expectation $E \circ F: \mathcal{M} \longrightarrow Z(\mathcal{N})$ satisfying $\tau \circ(E \circ F)=\tau \circ F=\tau$. Therefore there exist three orthogonal projections $e, f, g$ in $L^{2}(\mathcal{M}, \tau)$, respectively associated with $E, F, E \circ F$ such that $e f=g$. Thus we obtain ef $=f e$. Let us call

$$
\Delta=E+F-E F
$$

which a projection onto $\mathcal{M}_{E}+\mathcal{N}$ satisfying $\Delta\left(\mathcal{M}_{a h}\right) \subseteq \mathcal{M}_{a h}$. Then $\left.\Delta\right|_{\mathcal{M}_{a h}}$ projects onto $\left(\mathcal{M}_{E}+\mathcal{N}\right) \cap \mathcal{M}_{a h}=\operatorname{Lie}\left(\mathcal{N}_{E}\right)$ and extends to the orthogonal projection

$$
\left.\Delta\right|_{\mathcal{M}_{a h}}: L^{2}\left(\mathcal{M}_{a h}, \tau\right) \longrightarrow \overline{\operatorname{Lie}\left(\mathcal{N}_{E}\right)} \|^{\|},
$$

since $e$ commutes with $f$. Thus Theorem 3.8 applies. 


\subsection{An homogeneous space related to $C^{*}$-dynamical systems}

This example justifies in part Remark 3.10. Let $\mathcal{A}$ be a $C^{*}$-algebra with a faithful trace $\tau$ and $(\mathcal{A}, G, \alpha)$ a $C^{*}$-dynamical system. This means that $G$ is a locally compact group and $\alpha$ is a continuous homomorphism of $G$ into the $\operatorname{group} \operatorname{Aut}(\mathcal{A})$ of *-automorphisms of $\mathcal{A}$ equipped with the pointwise convergence topology. Let us assume that $G$ is compact and $\alpha$ is trace invariant, in the sense that

$$
\tau\left(\alpha_{t}(x)\right)=\tau(x)
$$

for all $t \in G$ and $x \in \mathcal{A}$. Consider the $C^{*}$-subalgebra of $\mathcal{A}$ given by

$$
\mathcal{A}^{G}=\left\{x \in \mathcal{A}: \alpha_{t}(x)=x, \forall t \in G\right\} .
$$

Denoting by $\mu$ the left Haar normalized measure on $G$, we can define

$$
E: \mathcal{A} \longrightarrow \mathcal{A}^{G}, E(x)=\int_{G} \alpha_{t}(x) d \mu(t) .
$$

It is apparent that $E$ is a norm one projection, and therefore a conditional expectation. We can easily show that $E$ is trace invariant:

$$
\tau(E(x))=\int_{G} \tau\left(\alpha_{t}(x)\right) d \mu(t)=\int_{G} \tau(x) d \mu(t)=\tau(x) .
$$

Then, the Lie algebra of the unitary group $U_{\mathcal{A}^{G}}$ which identifies with $\mathcal{A}_{a h}^{G}$ is closed and complemented. In proving that $U_{\mathcal{A}^{G}}$ is actually a Lie-Banach subgroup of $U_{\mathcal{A}}$, it remains to show that $U_{\mathcal{A}^{G}}$ is endowed with a structure of Banach-Lie group whose underlying topology is the norm topology of $U_{\mathcal{A}}$. By Proposition 4.4 of [6] it suffices to find an open neighborhood $V$ of $0 \in \mathcal{A}_{a h}$ and an open neighborhood $W$ of $1 \in U_{\mathcal{A}}$ such that the exponential map $\left.\exp \right|_{V}: V \longrightarrow W$ is a diffeomorphism and

$$
\exp \left(V \cap \mathcal{A}_{a h}^{G}\right)=W \cap U_{\mathcal{A}^{G}}
$$

Since the exponential map is the usual exponentiation of operators to prove the equation (4.1) we only have to note that if $u=e^{x} \in U_{\mathcal{A}^{G}}$ with $u$ close to 1 , $x \in \mathcal{A}_{a h}$, then $x \in \mathcal{A}_{a h}^{G}$. This follows since

$$
x=\log (u)=\sum_{n=1}^{\infty}(-1)^{n+1} \frac{(u-1)^{n}}{n},
$$

then

$$
\alpha_{t}(x)=\sum_{n=1}^{\infty}(-1)^{n+1} \frac{(u-1)^{n}}{n}=x .
$$

Thus $U_{\mathcal{A}^{G}}$ is a Lie-Banach subgroup of $U_{\mathcal{A}}$, so we can consider the homogeneous space $U_{\mathcal{A}} / U_{\mathcal{A}^{G}}$. Moreover, this is an orthogonal homogeneous reductive space since $E$ is trace invariant. 


\section{Acknowledgment}

The author wishes to express his gratitude to Professor Esteban Andruchow for suggesting the problem and for many helpful conversations during the preparation of the paper.

\section{References}

[1] E. Andruchow, L. Recht, Grassmannians of a finite algebra in the strong operator topology. Internat. J. Math. 17 (2006), no. 4, 477-491.

[2] E. Andruchow, G. Corach, M. Mbekhta, On the geometry of generalized inverses. Mathematische Nachrichten 278 (2005), no. 7-8, 756-770.

[3] E. Andruchow, L. Recht, Geometry of unitaries in a finite algebra: Variations formulas and convexity. Int. J. Math. (to appear).

[4] M. Argerami, D. Stojanoff, The Weyl group and the normalizer of a conditional expectation. Integral Equations and Operator Theory 34 (1999), no. 2, 165-186.

[5] M. Argerami, D. Stojanoff, Orbits of conditional expectations. Illinois J.Math. 45 (2001), no. 1, 243-263.

[6] D. Beltita, Smooth homogeneous structures in operator theory. Chapman and Hall/CRC, Monographs and Surveys in Pure and Applied Mathematics 137, 2006.

[7] C. Durán, L. Mata-Lorenzo, L. Recht, Natural variational problems in the Grassmann manifold of a $C^{*}$-algebra with trace. Adv. Math. 154 (2000), no. 1, 196-228.

[8] C. Durán, L. Mata-Lorenzo, L. Recht, Metric geometry in homogeneous spaces of the unitary group of a $C^{*}$-algebra. Part I: Minimal curves. Adv. Math. 184 (2004), no. 2, 342-366.

[9] C. Durán, L. Mata-Lorenzo, L. Recht, Metric geometry in homogeneous spaces of the unitary group of a $C^{*}$-algebra. Part II: Geodesics joining fixed endpoints. Integral Equations and Operator Theory 53 (2005), no. 1, 33-50.

[10] P. Halmos, J. McLaughlin, Partials isometries. Pacific J. Math. 13 (1963), no. 2, 585-596.

[11] L.A. Harris, W. Kaup, Linear algebraic groups in infinite dimensions. Illinois J. Math. 21 (1977), no. 3, 666-674.

[12] S. Kobayashi, K. Nomizu, Foundations of differential geometry. Vol. I. Reprint of the 1969 original, Wiley Classics Library, John Wiley \& Sons, 1996.

[13] L. Mata-Lorenzo, L. Recht, Infinite-dimensional homogeneus reductive spaces. Acta Cient. Venezolana 43 (1992), 76-90.

[14] G. Pedersen, $C^{*}$-algebras and their automorphism groups. Academic Press, 1979.

[15] A. Stacey, Variations on a theme: Riemannian geometry in infinite dimensions. Algebraic topology special session, BMC 2007.

[16] M. Takesaki, Theory of Operator Algebras I. Springer-Verlag, 1979. 
Eduardo Chiumiento

Dto. de Matemática

FCE-UNLP

Calles 50 y 115 (1900)

La Plata

Argentina

e-mail: eduardo@mate.unlp.edu.ar

Submitted: November 30, 2007.

Revised: June 15, 2008. 